\title{
Evaluations on Effectiveness of Bump-Slot over DAP-NAD-Based Tactical Wideband Wireless Networks*
}

\author{
Sewon Han and Byung-Seo Kim \\ Dept. of Computer and Information Communications Engineering, Hongik University, \\ 300 Shinan-ri, Jochiwon-ub, Yeongi-gun, \\ Chungcheongnam-do, Korea \\ hansewon@gmail.com, jsnbs@hongik.ac.kr
}

\begin{abstract}
MIL-SD-188-220 standard has specified the operations and functions of narrowband-based tactical networks. While the conventional tactical networks have mainly dealt with voice and short message data services, the future tactical networks are evolving towards broadband and multimediaenabled wireless Ad-Hoc networks. Therefore, this paper evaluates a network access method of the standard from the wideband network perspective, not narrowband perspective. In this paper, Deterministic Adaptable Priority Net Access Delay (DAP-NAD), one of the channel access methods specified in MIL-STD-188-220D with Change 1 standard, is simulated and evaluated over Wideband Networking Waveform-like environments in terms of Urgent precedence traffic. The simulation results conclude that unlike the narrowband environments, Bump-Slot, which is a unique time slot specified by the standard, does not play a critical role to enhance the performance in transmitting Urgent traffic over the wideband networks.
\end{abstract}

Keywords: MIL-STD-188-220, Tactical networks, DAP-NAD, Bump-Slot.

\section{Introduction}

MIL-STD-188-220 standard specifies physical, data link, and intranet protocol layers for tactical communication devices using narrowband channels [1]. The standard has been versioned up from MIL-STD-188-220A to MIL-STD-188-220D with Change 1 (Hereinafter, MIL-STD-188-220 standard means MIL-STD-188-220D with Change 1 throughout the paper). In general, main traffic over tactical networks are voices and short messages. However, because the future tactical environment is moving towards network-centric warfare, the tactical communications and networks are also evolved to obtain and to utilize various types of tactical information through multimedia traffic including voice, video, and a large-sized data traffic over wideband

\footnotetext{
* This research was supported in part by the National Research Foundation of Korea (NRF) grant funded by the Korea government (MEST) (2011-0005360) and in part by the MKE (The Ministry of Knowledge Economy), Korea, under the ITRC (Information Technology Research Center) support program supervised by the NIPA (National IT Industry Promotion Agency" (NIPA-2011- (C1090-1121-0011)).
} 
communication channels. Therefore, the networks provide the better awareness on the current battlefield situations and as a consequence, the better command and control are achieved. Furthermore, the network topologies are evolving to be flexible and scalable without any infrastructure and centralized nodes like mobile ad-hoc networks. In the summary, unlike narrowband infrastructure-based conventional tactical networks in the past, the future tactical networks have been evolved as wideband wireless ad-hoc networks with multimedia services.

This trend of the future tactical networks is proved by Joint Tactical Radio Systems (JTRS) which has been planned to be the next-generation voice-and-data radio used by the U.S. military in the field operations [2]. JTRS is a wideband mobile ad-hoc network with a software-defined radio technology to work with various military and civilian radio. Fig. 1 shows tan overview of JTRS network architectures [3]. JTRS specifies several types of waveforms. One of the forms is Wideband Networking Waveform (WNW). WNW has been proposed and developed to provide the wider channel bandwidth and the higher data rate to the tactical wireless networks [4]. Based on the specification provided by Spectrum company in [4], WNW support the high data rates from $100 \mathrm{Kbps}$ to 23Mbps using OFDM-based physical layer over 1.2 $\sim 10 \mathrm{MHz}$ bandwidths as shown in [4]. Besides, Software Defined Radio (SDR) and Cognitive Radio (CR) are also extensively researched for the opportunistic communications over scarce frequencies. While the efforts to enhance the physical layer for the tactical networks has mainly focused in the past decade such as WNW, $\mathrm{SDR}$, and CR, the data link protocol to efficiently deliver the data to multi users is not extensively studied.

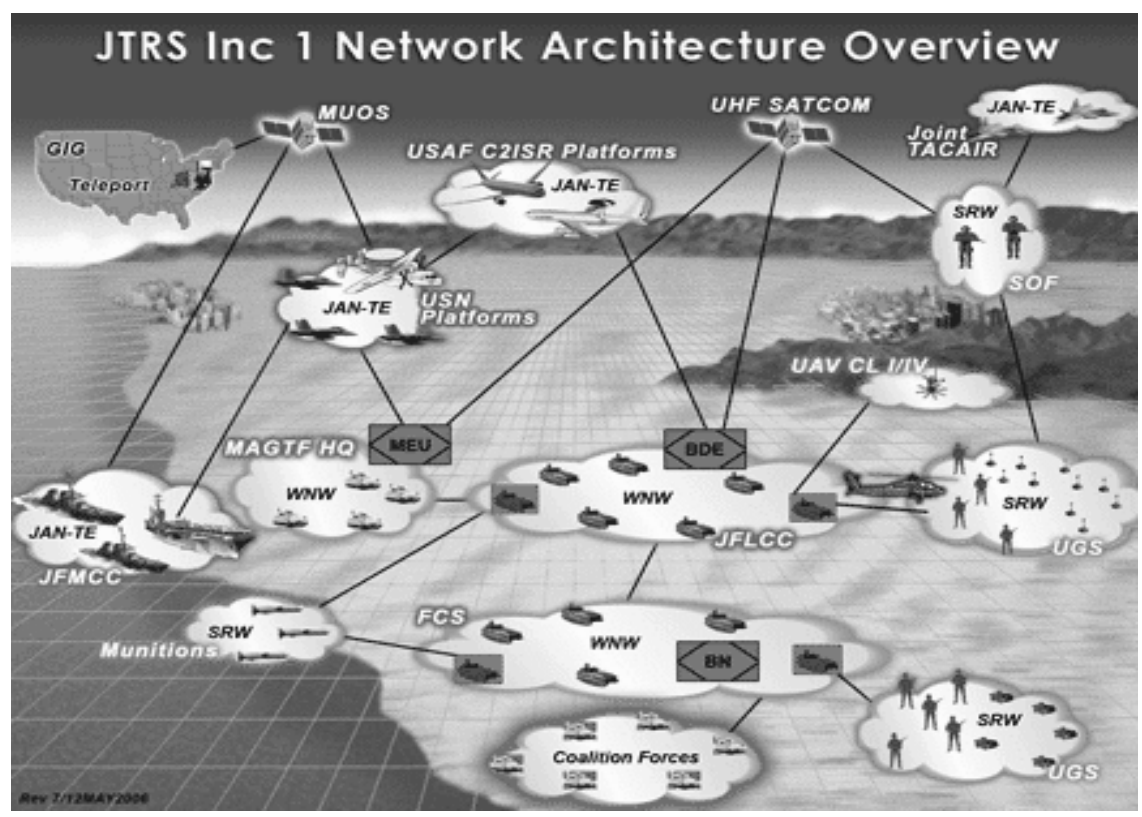

Fig. 1. An overview of JTRS networks architecture [3] 
The researches in the past regarding the data link protocols based on different versions of MIL-STD-188-220 standards have used the network parameters for oldtype tactical communication environments, not for the future environment such as wide bandwidth, high data rate, and multimedia traffic types [5]-[12]. Recently, [13] has compared two of 6 channel access methods specified in MIL-STD-188-220 standard to Carrier Sense Multiple Access and Collision Avoidance (CSMA/CA) protocol over IEEE802.11a-based physical layer which is $20 \mathrm{MHz}$ wideband channel. The two methods of MIL-STD-188-220 standard are Random net access delay (RNAD) and Deterministic adaptable priority net access delay (DAP-NAD). The research shows that while R-NAD has worse performance than CSMA/CA protocol has, DAP-NAD has better performance than CSMA/CA protocol has. However, the paper does not analyze the behavior of the DAP-NAD method itself over the sideband systems.

Even though the channel access methods in MIL-STD-188-220 standard are designed for the old-fashioned narrowband systems, it is needed to evaluate the methods for the future tactical wideband systems. The reason is because using the methods over the WNW may have advantages in terms of reducing the cost to develop new protocols, providing interoperability new communication devices with the old devices, and simplifying communication specifications over all tactical communication devices.

Therefore, this paper evaluates the DAP-NAD channel access method over the wideband network scenarios and clams a issue using a Bump-Slot which is a time slot for a specific purposes mentioned in Section 2.2.

In this paper, Section 2 introduces the specifications on the data link protocols specified in MIL-STD-188-220 standard and reviews the previous researches regarding on the protocol. Particularly, one of medium access control (MAC) methods in the standard is illustrated in details. Furthermore, a potential issue is claimed. In Section 3, DAP-NAD method is simulated and the claimed issue is evaluated through the simulation results. Finally, the conclusions are made in Section 4.

\section{MIL-STD-188-220D with Change 1}

\subsection{Network Access Control (NAC)}

MIL-STD-188-220 standard defines NAC to arbitrate the transmissions of multiple communication nodes. NAC is composed of 4 functions; Net busy sensing, Response Hold Delay (RHD), Timeout Period (TP), and Network Access Delay (NAD). Net Busy Sensing is a way to detect a signal over the channel, RHD defines the times from the data transmission to the reception of an acknowledgement packet, and TP defines a waiting time of a node before transmitting a data. NAD defines how many slot-times a node has to wait before making its transmission and how to act before its transmission after TP is expired.

MIL-STD-188-220 standard defines 6 different NADs including Random-NAD (R-NAD), Prioritized-NAD (P-NAD), Hybrid-NAD (H-NAD), Radio EmbeddedNAD (RE-NAD), Deterministic Adaptable Priority NAD (DAP-NAD), and Data And Voice NAD (DAV-NAD). Moreover, MIL-STD-188-220 standard defines three 
precedences for the traffic: Urgent, Priority, and Routine precedence. Urgent precedence is the highest precedence and Routine precedence has the lowest precedence. P-NAD, DAP-NAD, and DAV-NAD are designed to provide the better transmission opportunities to the higher precedence traffic.

The paper in [5] compares R-NAD with P-NAD in terms of the average network access delay and the collision rate with varying the packet size and load balancing. The paper concludes R-NAD has better performance than P-NAD does. Researches in [6] and [7] shows DAP-NAD has better performance than RE-NAD has in terms of the end-to-end delay and the network utilization. In addition, in [8], DAP-NAD is compared with DAV-NAD in terms of the latency and the network utilization and concludes DAP-NAD provides the better performance. As a consequence of aforementioned previous researches, this paper focuses on DAP-NAD method among 6 NADs.

\subsection{DAP-NAD}

In DAP-NAD method, nodes access a medium and transmit their data in a prescheduled its time slot like TDMA system, but the length of time slot is varied whose size is the same as the packet length. The transmission order is re-scheduled after the completion of one sequence. However, unlike TDMA system, there are unique characteristics in DAP-NAD as follows.

First of all, the transmission order is not decided by a central scheduler, but by nodes themselves. Participating nodes are assigned with a unique Identification (ID) number when nodes join the networks and the ID itself defines the transmission order using the round-robin manner. For example, if there are 3 nodes and their IDs are 1, 2, and 3, then the transmission order of nodes is 1,2, and 3. All nodes have their slots in a round. If one node with ID 1 transmits its own time slot, then the next round starts with node with ID 2 and the sequence is 2,3 , and 1 .

Secondly, as aforementioned above, each packet has one of the three precedences. Each transmission sequence also has a network-precedence, which means that only a node having a packet with same precedence as the current network-precedence can transmit during its own time slot. Node can transmit its packet at its turn only if the precedence of its pending packet is the same as or higher than the networkprecedence. During a round of the transmission sequence, if there is no transmission with the current network-precedence, then the network-precedence of the next round sequence is downgraded. For example, let's assume that the network-precedence of the first round is Urgent. If there is no Urgent packet transmissions during the transmission round, then network-precedence is Priority that means node having Urgent or Priority precedence can send their packet in their time slot in that round. However, if there is transmission with the precedence the same as the networkprecedence, then the current network-precedence is maintained for the next round.

Thirdly, there is unique time slot, called Bump-Slot. The slot follows right after any slot in which any actual transmission completes. Any node having only an Urgent packet can transmit its packet during the Bump-Slot regardless of its transmission turn only if the current network-precedence is not Urgent. If there is any transmission in the Bump-Slot, the network-precedence for the next round is updated to Urgent. Therefore, the Bump-Slot is used to give more transmission opportunity to a node 
with Urgent packet, so that it improves the performance of Urgent packet. Like TDMA-based medium access, in general, the transmission is performed in the contention-free manner. However, the transmission during the Bump-Slot is based on slotted aloha medium access. That is, collisions occur only during Bump-Slot in DAP-NAD.

An example of DAP-NAD channel access method is illustrated in Fig. 2.

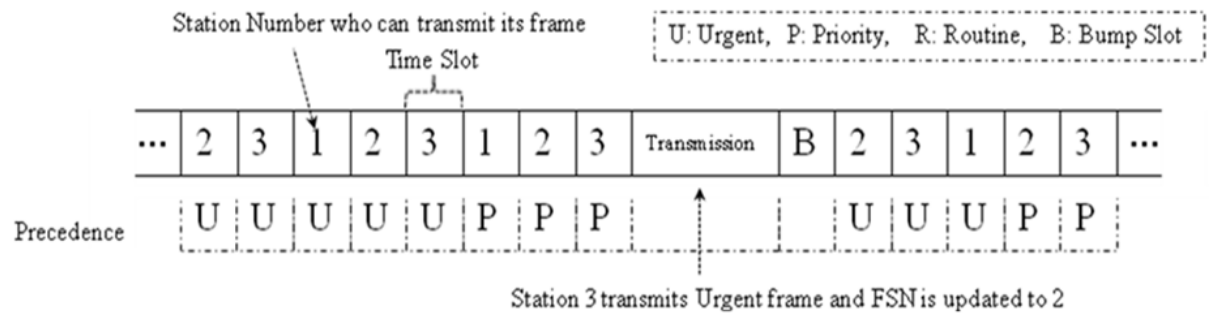

Fig. 2. An example of DAP-NAD access method

\subsection{Motivations}

As mentioned before, the channel access method in Bump-Slot is a slotted aloha method, so that it causes collisions among nodes having Urgent packets. The collision in the slot generally increases as the number of nodes with the Urgent packets increases. The conventional tactical communication devices using DAP-NAD method has been operating with the low data rates over the narrow bandwidth channels. The low data rate requires relatively longer transmission time than the high data rate over the wide bandwidth channel does. In addition, this makes a slot time longer because the duration of each time slot in DAP-NAD is the same as the duration of packet transmission time unlike constant time slots in TDMA-based cellular networks. Therefore, over the communication environments of the conventional tactical networks, increasing the number of nodes prolongs the waiting time of nodes that are waiting their reserved time slots. Such a long waiting time might be critical for the Urgent packet transmissions which has a delay constraint. To prevent from this, the Bump-Slot resets the network-precedence to Urgent. Therefore, the nodes with Urgent packets have more transmission opportunities so that it reduces the transmission delay of the Urgent packet. That is, the Bump-Slot is essential to enhance the performance of Urgent packet transmissions over the narrow bandwidth channels.

On the other hand, the future tactical networks are operating over the wide bandwidth channels. Thus, it might be considerable if the Bump-Slot is also effective over the wide-bandwidth channel. Over the wideband networks, the transmission time is reduced due to the high data rates and as a consequence the transmission delay is shortened. In addition, the time to take that a node waits its scheduled time slot becomes much shorter in the wideband networks than that in the narrow-bandwidth channels. Therefore, the Bump-Slot might not be necessary because the transmission delays of Urgent packets over the wide-bandwidth channels may not be so bad. Besides, because the Bump-Slot causes collisions and there is more urgent traffic over 
the wideband system, it may make the performance of the Urgent packet worse. Therefore, in the next section, the performance of DAP-NAD with the Bump-Slot is compared to that of the DAP-NAD without the Bump-Slot over wideband channel environments throughout the extensive simulations.

\section{Simulations and Evaluations}

\subsection{Simulation Environments}

In this section, the effectiveness of the Bump-Slot over the wideband networks is evaluated through the extensive simulations. Note that the objective of this paper is to evaluate the only channel access mechanism over wideband networks with multimedia traffic. In addition, it is also hard to obtain exact network parameters for the wideband tactical networks due to confidentiality. Therefore, the simulation uses some parameters that can be obtained from the public resources and assumed to be similar to the future wideband tactical networks.

For this evaluations, it is assumed that DAP-NAD method operates over Orthogonal Frequency Division Multiplexing (OFDM)-based WNW illustrated in [3] as a physical layer. Because the exact specification of WNW is confidential and is not revealed in the public, the information on the possible data rates and bandwidths for WNW is obtained from the open source such as [3]. For the simulations, the data rate is set to $3 \mathrm{Mbps}$ with $10 \mathrm{MHz}$ bandwidth. Furthermore, the well-known OFDM-based wireless data network is IEEE802.11-based wireless local area networks (WLANs). Thus, the network parameters for the simulations are adopted from in IEEE 802.11a standard [14]. Because the paper focuses on the performance of channel access method itself, the parameters including frame format, processing time, propagation delay (which is set to 0 ), the sizes of preamble and header and so on uses those of IEEE802.11a unlike system parameters used by [5]-[12] and MIL-STD-188-220 standard. On the other hand, because the lowest data rate of IEEE802.11a standard is $6 \mathrm{Mbps}$ with $20 \mathrm{MHz}$ bandwidth and the simulation uses $3 \mathrm{Mbps}$ rate with $10 \mathrm{MHz}$ bandwidth, most of parameters in IEEE 802.11a are expanded two times longer than the original values. For instance, the duration of Short InterFrame Space (SIFS) is doubled comparing to the value specified in IEEE 802.11a.

Table 1. Simulation parameters

\begin{tabular}{|l|l|}
\hline \multicolumn{1}{|c|}{ Parameter } & \multicolumn{1}{c|}{ Value } \\
\hline Data Rate & $3 \mathrm{Mbps}$ \\
\hline Preamble & $32 \mathrm{us}$ \\
\hline Physical layer header & $16 \mathrm{us}$ \\
\hline MAC header & $272 \mathrm{bits}$ \\
\hline Default Slot Time & $18 \mathrm{us}$ \\
\hline ACK packet & $88 \mathrm{us}$ \\
\hline SIFS & $32 \mathrm{us}$ \\
\hline
\end{tabular}


Default Slot Time in the Table 1. indicates the minimum time duration taken to decide if the channel is busy and it is set to the same time as backoff slot time in IEEE 802.11a. That is, if no transmission is detected during the time, the next time is automatically started. There is SIFS between the end of transmitted packet and the beginning of the next time slot.

The detailed parameters used in the simulation are shown in Table 1.

The error-free channel is used for the simulations. The number of nodes is set to 100 and it is assumed that all nodes are in one-hop radio range, so that all nodes can hear one another. The total number of nodes in the simulation is 100 . The number of nodes generating Urgent, Priority and Routine precedence traffic are set to 30, 35, and 35 nodes, respectively. All nodes generate their packets with the exponential distribution with 500 byte-average length and with Poisson-distributed inter-arrival time with $1 / \lambda$ average time. The lifetime of the packet at the MAC layer is set to $150 \mathrm{~ms}$ which is recommended in International Telecommunication Union (ITU) as shown in [15][16]. As a performance metrics, the average End-to-End delay, the average Packet Delivery Ratio (PDR), and the average throughput are measured at the MAC layer.

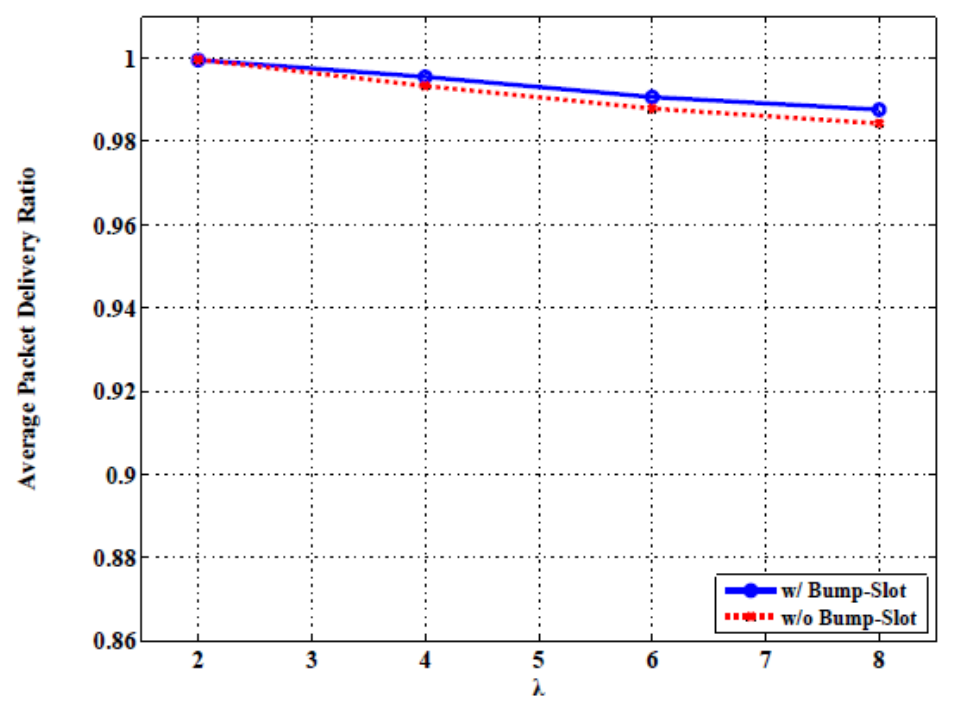

Fig. 3. Average packet delivery ratio of Urgent precedence traffic as a function of $\lambda$

\subsection{Performance Evaluations}

The average packet delivery ratios and end-to-end delays of DAP-NAD with BumpSlot, named "w/ Bump", and without Bump-Slot, named "w/o Bump", as $\lambda$ increases are shown in Fig. 3 and Fig. 4, respectively. The average end-to-end delay takes into account only the delays of successfully transmitted packets, not the dropped packets. The performances are only for Urgent traffic. The performance of the two methods are similar each other cross over all values of $\lambda$. 
Regarding packet delivery ratio, both of methods provides almost same performance as shown in Fig. 3. The differences of the performance of both methods are less than $1 \%$. On the other hand, the average packet delay of the method with Bump-Slot shows the $40 \%$ higher than that of one without Bump-Slot. However, the average end-to-end delays of both methods are much less than $150 \mathrm{~ms}$ requirements specified in [15] and [16]. Therefore, in terms of the delay requirement, even the relative high delay of the method without Bump-Slot is acceptable.

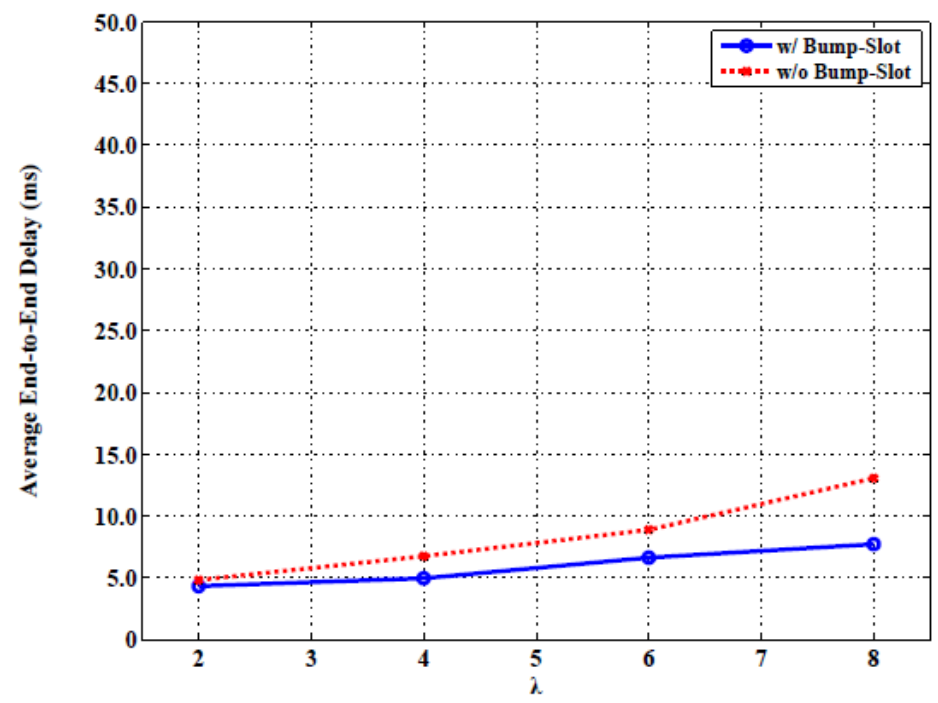

Fig. 4. Average end-to-end delay of Urgent precedence traffic as a function of $\lambda$

Fig. 5 shows the average packet delivery ratios of Priority and Urgent traffic using DAP-NAD with Bump-Slot and without Bump-Slot as $\lambda$ increases. The performance of the both methods is almost similar, but the performance of DAP-NAD without Bump-Slot has better performance than that of one with Bump-Slot. The results are clear because the role of Bump-Slot increases only the performance of the Urgent traffic by scarifying the other traffic. However, as shown in Fig. 3 and 4, the improvement of the Urgent traffic is hardly seen.

In Fig. 6, the average Bump-Slot Utilization and the collision rate in Bump-Slots are shown. As shown in the figure, the utilization of Bump Slots decreases after $\lambda$ is 6 . The reason of this decrease is that even though Bump-Slots are generated after all transmissions, no node sends its packet because the network-precedence of the most transmission round is Urgent. As the standard [1] specifies, Bump-Slot is uses only when the network-precedence is lower than Urgent. In addition, the Collision ratio increases as the traffic increase and it deteriorates the performance of Urgent transmissions.

As results, the overall performance of DAP-NAD with Bump-Slot is hardly improved comparing to one without Bump-Slot because of the increase of collisions in the slots and the low utilization of Bump-Slots. 


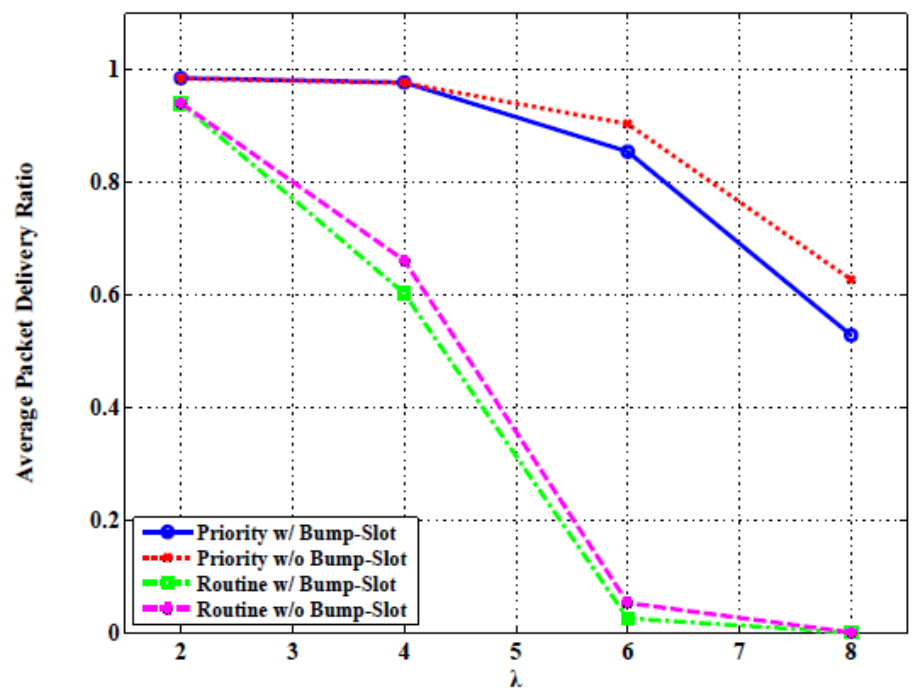

Fig. 5. Average packet delivery ratio of Priority and Routine precedence traffic as a function of $\lambda$

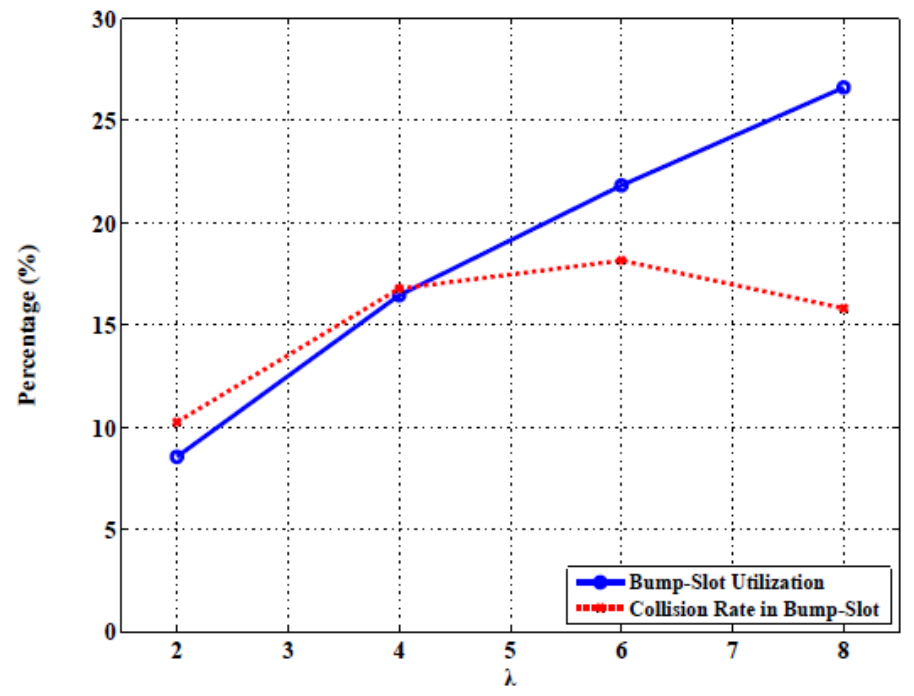

Fig. 6. Average Bump-Slot utilization and collision rate in Bump-Slots as a function of $\lambda$

\section{Conclusions}

The future wireless tactical networks are evolving to serve wideband multimedia traffic. MIL-STD-188-220 standard specifies the channel access method over the voice-based narrowband networks. Using the methods in the future tactical networks 
provides some advantages in terms of providing the interoperability with the legacy systems and the simplicity on the development of the future systems. In this paper, one of channel access method specified in MIL-STD-188-220, named DAP-NAD, is evaluated over wideband environment and points out the problem of using BumpSlot. The Bump-Slots resets the network-precedence to Urgent to give more transmission opportunity to the Urgent traffic. However, over the wideband environments, using the Bump-Slots hardly improves the performance of DAP-NAD networks because of many collisions and the low utilizations on the Bump-Slots. Therefore, this paper claims the effectiveness of Bump-Slots in DAP-NAD method is lost over the wideband networks and there are new methods to improve the performance of Urgent traffic because of the importance of the traffic in the battlefield.

\section{References}

1. MIL-STD-188-220D with Change1 Digital Message Transfer Device Subsystem (June 23, 2008)

2. JPEO JTRS Standard, http: / /sca.jpeojtrs.mil

3. Abacus Programming Corporation, http://www.abacuscorp.com/se.htm

4. Spectrum Signal Processing by Vecima, http: / /www. spectrumsignal.com/ products/pdf/wnw_starter_kit.pdf

5. Burch, R.G., Chamberlain, S.C.: Performance evaluation of MIL-STD-188-220 interoperability standard for digital message transfer device subsystems. In: 14th Military Communications Conference, pp. 427-432 (1995)

6. Thuente, D.J., Borchelt, T.E.: Simulation studies of MAC algorithms for combat net radio. In: 16th Military Communications Conference, pp. 193-199 (1997)

7. Thuente, D.J., Borchelt, T.E.: Simulation model and studies of MIL-STD-188-220A. In: 17th Military Communications Conference, pp. 198-204 (1998)

8. Thuente, D.J., Borchelt, T.E.: Efficient data and voice media access control algorithm for MIL-STD-188-220B. In: 19th Military Communications Conference, pp. 115-121 (2000)

9. Thuente, D.J., Whiteman, J.K.: Modified CSMA/Implicit token passing algorithm for MIL-STD-188-220B. In: 20th Military Communication Conference, pp. 838-844 (2001)

10. Thuente, D.J.: Improving quality of service for MIL-STD-188-220C. In: 21st Military Communication Conference, pp. 1194-1200 (2002)

11. Yang, J., Liu, Y.: An improved implicit token passing algorithm for DAP-NAD in MIL-STD188-220C. In: 2nd International Conference on Wireless Communications, pp. 1-4 (2006)

12. Liu, Y., An, J., Liu, H.: The modified DAP-NAD-CJ algorithm for multicast applications. In: 4th International Conference on Wireless Communications, Networking and Mobile Computing, pp. 1-4 (2008)

13. Kim, B.-S.: Comparative Study of MIL-STD-188-220D Standard over IEEE802.11 Standard. SK Telecommunications Review 20(1) (2010)

14. Part 11: Wireless LAN Medium Access Control (MAC) and Physical Layer (PHY) specifications, IEEE Std. 802.11 (June 12, 2007)

15. Calyam, P., Sridharan, M., Mandrawa, W., Schopis, P.: Performance Measurement and Analysis of H.323 Traffic. In: Barakat, C., Pratt, I. (eds.) PAM 2004. LNCS, vol. 3015, pp. 137-146. Springer, Heidelberg (2004)

16. International Telecommunication Union, http://www.itu.int/itudoc/itu-t/workshop/qos 